\title{
70. SEISMIC EVIDENCE OF ANISOTROPY IN THE YAMATO BASIN CRUST
}

\author{
Naoshi Hirata, ${ }^{2}$ Hiroki Nambu, ${ }^{2}$ Masanao Shinohara, ${ }^{2,4}$ and Kiyoshi Suyehiro ${ }^{3}$
}

\begin{abstract}
A seismic anisotropy was found in the upper crust of the northern Yamato Basin, Japan Sea, during the downhole experiment in 1989. An ocean broadband downhole seismometer (OBDS) and nine ocean bottom seismometers (OBSs) were installed during Ocean Drilling Program (ODP) Leg 128 in and near Hole 794D. Traveltimes of $P$-wave recorded by the OBDS, and nearest three OBSs, which were corrected due to offset ranges from a shot to the receiver and thicknesses of the sedimentary layer, were fitted by an anisotropic velocity model: the azimuthal dependence of the velocity of $P$-wave propagation is expressed in terms of sinusoidal functions up to the sixth-order terms. Both the anisotropic terms, the second- and the fourth-order terms, and the laterally heterogeneous term, the first-order term, were significant in the model. The direction of $P$-wave propagation was measured by the OBS array data and found to deviate from the sagittal plate. The traveltimes and the direction of propagation both indicate that $P$-wave propagates faster east-west than north-south by $4 \%-7 \%$. The anisotropy in the basement layer is interpreted to be due to stress induced opening of cracks.
\end{abstract}

\section{INTRODUCTION}

Since Stephen (1981) reported observations of seismic anisotropy from borehole data in the western Atlantic, there have been several studies that indicate evidence of anisotropy in the upper oceanic crust (Stephen, 1981, 1985; White and Whitmarsh, 1984; Little and Stephen, 1985; Shearer and Orcutt, 1985). Although seismic anisotropy in oceanic layer 2 seems to be the most probable explanation of azimuthal dependence of wave propagation, it is sometimes hard to distinguish between anisotropy and lateral heterogeneity from seismic data. Moreover, it is also likely that both anisotropy and lateral heterogeneity contribute to complexity of wave propagation. For example, Stephen (1988) reported the model with a horizontal velocity gradient of $2 \mathrm{~s}^{-1}$ in the same area that Stephen (1981) investigated anisotropy. So, it is important to conduct an experiment with well-controlled geometry to investigate the dependence of wave propagation on directions.

The accuracy of locations of the seismic sources and receivers is one of the essential points for traveltime analysis. Navigation with the Global Positioning System (GPS) enables us to have good control of ship position. Seismometers in the deep sea drilling hole could be an excellent reference for locations of the ship from which a controlled seismic source was fired. A dense array of seismometers on the ocean bottom and reflection data can constrain the variation of local structure in the crust.

In 1989 a seismic experiment in Ocean Drilling Program (ODP) Hole 794D in the northern Yamato Basin, northeast Japan Sea, was conducted for a detailed study of crustal structure and anisotropy. The Japan Sea is one of the marginal basins in the northwestern Pacific. The sea has two major basins: the Japan Basin and the Yamato Basin, which are divided by the Yamato Rise. The Japan Basin has an oceanic crustal structure, and the Yamato Rise has a thick crust in which seismic velocity is comparable to that of a continental crust (Murauchi, 1972; Ludwig et al., 1975). The southern part of the Yamato Basin is neither a typical ocean nor a continent (Hirata et al., 1987, 1989; Katao, 1988; Chung et al., 1990). The structure of the Yamato Basin was investigated by a modern method using an ocean bottom seismographic array. The crustal structure of the Japan Sea is by no means simple and homogeneous. Moreover, a seismic anisotropy in

\footnotetext{
I Tamaki, K., Suyehiro, K., Allan, J., McWilliams, M., et al., 1992. Proc. ODP, Sci. Results, 127/128, Pt. 2: College Station, TX (Ocean Drilling Program).

${ }^{2}$ Department of Earth Sciences, Faculty of Science, Chiba University, 1-33 Yayoicho, Chiba 263, Japan.

${ }^{3}$ Ocean Research Institute, University of Tokyo, Tokyo 164, Japan.

${ }^{4}$ Present address: Ocean Research Institute, University of Tokyo, Tokyo 164, Japan.
}

the uppermost mantle is also reported (Okada et al., 1978; Chung, 1992). There has been, however, no attempt to detect an anisotropy in the crust of the Japan Sea so far.

We will here report evidence of seismic anisotropy in the upper crust using an ocean broadband downhole seismometer (OBDS) and an array of ocean bottom seismometers (OBSs). Instrumentation and installation of the OBDS is reported by Suyehiro et al. (this volume) and the detailed crustal structure by Shinohara et al. (this volume). The latter study focuses on the lateral heterogeneity of the crust. We will concentrate on the seismic anisotropy in the present report.

\section{EXPERIMENT}

In order to study the crustal seismic anisotropy we conducted an air gun profiling experiment using a downhole and ocean bottom seismographic array in the northern part of the Yamato Basin. We deployed a three-component ocean broadband downhole seismometer (OBDS) at Hole 794D of the Ocean Drilling Program (Ingle, Suyehiro, von Breymann, et al., 1990) and an ocean bottom seismographic (OBS) array around the hole (Fig. 1). We conducted a reflection/refraction experiment using an air gun as a controlled seismic source. The OBDS was installed and clamped at a depth of $714.5 \mathrm{~m}$ below seafloor (mbsf) by the JOIDES Resolution. Details of instrument and installation are described in Suyehiro et al. (this volume). The OBS array was deployed by the Tansei-maru, consisting of nine OBSs on two mutually perpendicular lines. Among the OBSs five were distributed with an interval of $2800 \mathrm{~m}$. One of the OBSs, JRT4, was located in the close vicinity of the hole (Fig. 2). Hence, the OBDS and five OBSs formed a three-dimensional small-aperture seismic array. We will use the data from the OBDS and the inner three OBSs, JRT3, JRT4, and JRT6 in the array for the anisotropy study.

We fired two 9-liter air guns or one 17-liter air gun on two linear lines and two circles. Data from the circle profiling will be used in the present study to investigate the azimuthal dependence of wave propagation in the crust. The inner shooting circle has a radius of $9 \mathrm{~km}$ and the outer has a radius of $18 \mathrm{~km}$. The two-dimensional crustal structure near Hole 794D was derived from the data of the linear profiling as reported by Shinohara et al (this volume).

The distances between sources and receivers of the seismic signals are essential to discussing azimuthal variations of traveltimes due to the anisotropy. We use the Global Positioning System (GPS) as a primary data of the position of the shooting ship. The GPS navigation data were available about $80 \%$ of the operational period. During the period in which the GPS was unavailable, the LORAN-C data were used after smoothing and correcting with respect to the GPS coordi- 


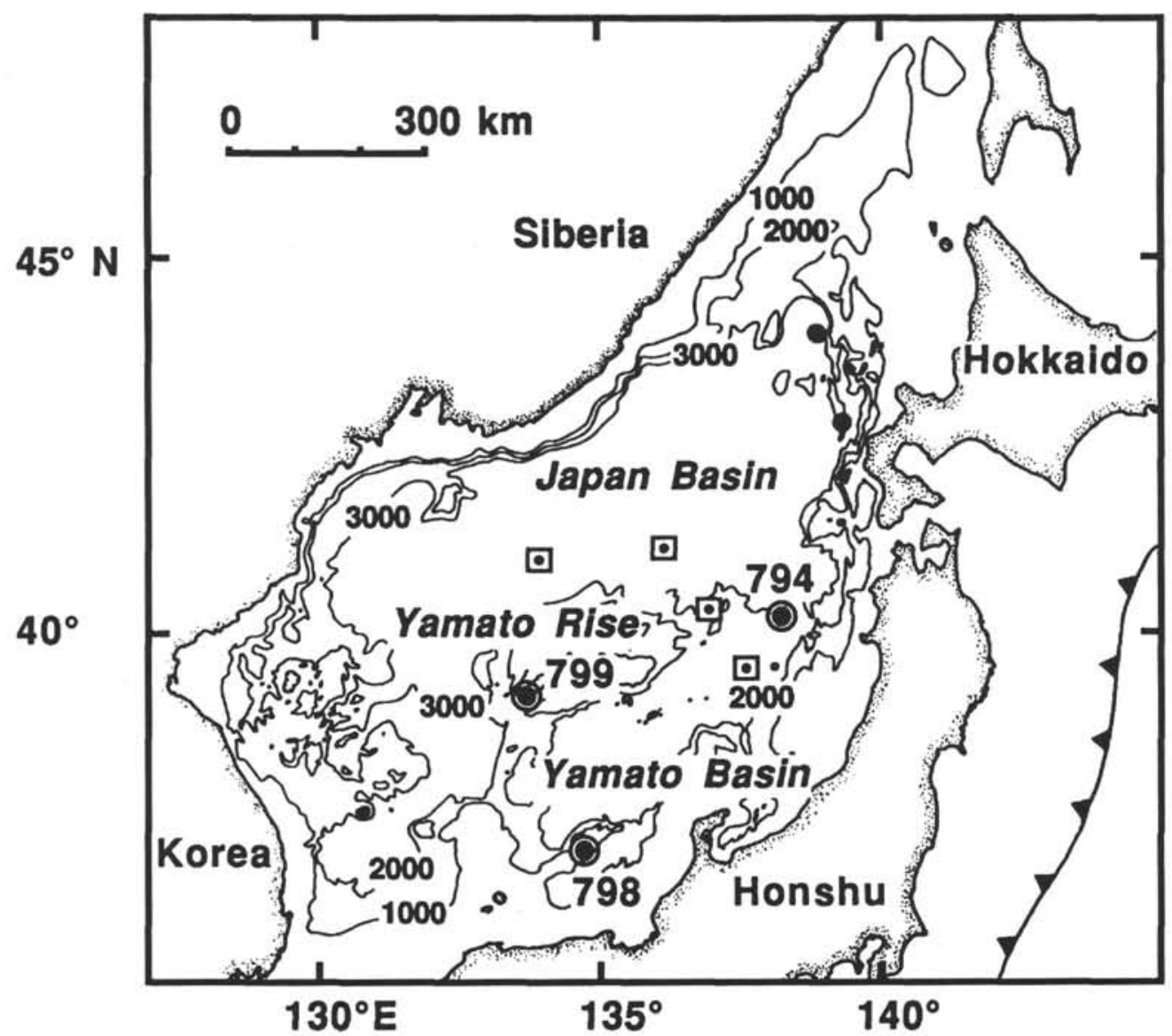

Figure 1. Map showing the Japan Sea (after Ingle, Suyehiro, von Breymann, et al., 1990). Circled dots denote Leg 128 Sites 794, 798, and 799. Dots show Leg 127 Sites 794 through 799. Boxed dots indicate DSDP Sites 299 through 302. Bathymetry in meters.

nate. The navigation data were acquired continuously with sampling intervals between $4 \mathrm{~s}$ and $15 \mathrm{~s}$. We found a systematic difference between the GPS and LORAN-C positions by about $300 \mathrm{~m}$, which varies during the observational period. We estimated the differences during the period in which the GPS was unavailable by interpolating or extrapolating the differences in GPS available period. The hybrid shot positions are shown in Figure 3. The position of Hole 794D was well located by the GPS and was used as the reference position for all other shots and OBSs. Since we used the Tokyo datum as the horizontal geodetic datum, we converted all GPS fixed positions in WGS84 into those in Tokyo datum. The OBSs were relocated by using direct water waves of air gun signals. Since the geometry of the shot and receiver positions is very good, we could locate OBSs very precisely. The error in the OBS position is estimated to be about $5 \mathrm{~m}$ in a least squares sense, provided that the error in traveltimes is $0.1 \mathrm{~s}$. The root mean square of the residuals in traveltimes for the relocated OBS, for example, JRT4, is about $0.03 \mathrm{~s}$. So we think that the total errors in locations of OBSs with shot positions are less than $50 \mathrm{~m}$. The positioning data of the GPS have random errors of about $20 \mathrm{~m}$, which is estimated from the scattering of the continuous positioning data. The drift of the clocks installed in the OBSs and that of the air gun shooting system aboard is calibrated with respect to the Japan Standard Time through the radio signal (JJY) before launching and after retrieval of the OBSs. The clocks were maintained with an accuracy of $0.05 \mathrm{~s}$ or better during the air gun operation.

Single channel seismic (SCS) reflection survey was simultaneously conducted during the air gun-OBS profiling. The reflection data were acquired on the Tansei-maru in digital form. We recorded exactly the same air gun shooting by the OBDS, OBSs, and a hydrophone streamer.

\section{DATA}

Our basic data-sets include the traveltimes of the first arrivals, offset distances, and two-way traveltimes down to the acoustic basement from the single channel reflection profiles on the inner and outer circle shootings.

We show an example of seismograms for the inner circle shooting recorded by the OBDS in Figure 4. The first arrival phases correspond to waves traveling through the uppermost basement layer with an average $P$-wave velocity of $4.5 \mathrm{~km} / \mathrm{s}$ (Fig. 5). Although the topography is relatively smooth in the studied area, the thickness of the sedimentary layer varies from site to site (Fig. 6). We manually picked the first arrivals from the refraction seismograms and two-way traveltimes to the acoustic basement from the reflection profiles. An example of the picked traveltimes is shown in Figure 7 in comparison with offset distances from the shot positions to the receiver, and two-way traveltimes down to the acoustic basement at the position where the ship shot the air gun. The data show that traveltimes vary between 4 and $5 \mathrm{~s}$. The overall variation of the traveltimes is well correlated to that of offset distances. Since the $P$-wave velocity in the basement is $4.5 \mathrm{~km} / \mathrm{s}$, the variation in the offset by $5 \mathrm{~km}$ changes the traveltime more than $1 \mathrm{~s}$. Data from JRT4, located above the OBDS, are shown in Figure 8 and those from JRT3 and JRT6 are in Figures 9 and 10 , respectively.

The refraction arrivals from the outer circle shooting traveled in the upper part of the $6.2 \mathrm{~km} / \mathrm{s}$ layer as shown in Figure 5 (Shinohara 


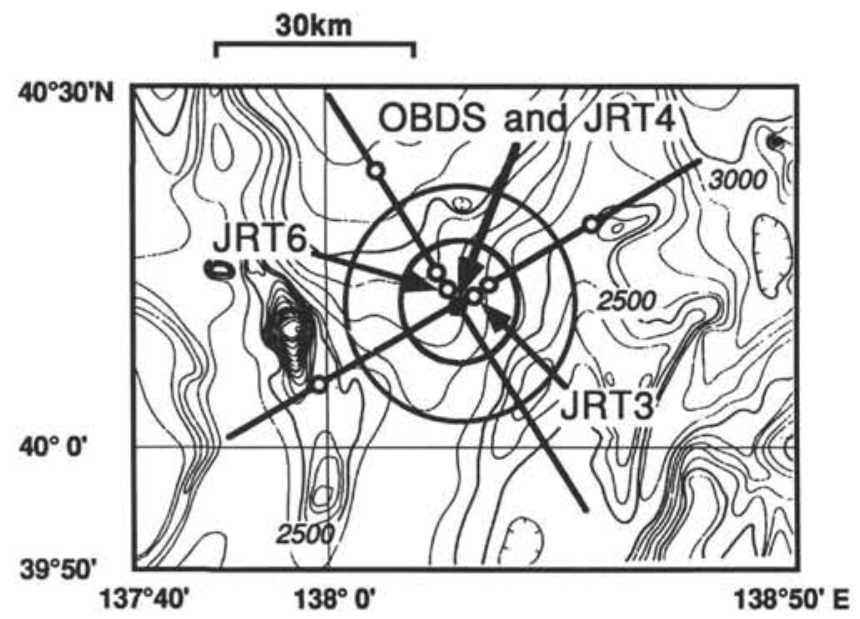

Figure 2. Location map showing the OBDS and OBS array. Dot denotes Hole $794 \mathrm{D}$, at which the OBDS and JRT4 were deployed. Circles show OBSs. Bathymetry in meters. The contour interval is $100 \mathrm{~m}$. Air gun profiling was conducted along two mutually perpendicular lines and two circles (solid lines).

et al., this volume). The signal-to-noise ratio of the first arrival phase is not as good as that of the inner circle shooting data (Fig. 11). We show the traveltimes of the refracted wave, offset distances, and the two-way traveltimes down to the acoustic basement from the sea surface (Fig. 12).

\section{TRAVELTIME ANALYSIS}

\section{Method}

Traveltimes are modeled by the following formula:

$$
t_{i}=l_{i} / v(\theta)+t_{0}+\kappa t_{b i},
$$

where $t_{i}$ is a traveltime of the refraction arrival for the $i$-th shot, $l_{i}$ is an offset distance between the $i$-th shot position and the receiver, $v(\theta)$, is a velocity in the layer in which the refracted wave travels, $t_{0}$ is a time term of receiver, $t_{b i}$ is one-way traveltime down to the acoustic basement for the $i$-th shot, and $\kappa$ is a coefficient of $t_{b i}$. If the receiver is located on the interface beneath which the refracted wave propagates, then $t_{0}$ is zero, which is the case for the OBDS receiving the inner circle shooting. For the outer circle shooting recorded by the OBDS, $t_{0}$ is a time term of the $4.5 \mathrm{~km} / \mathrm{s}$ layer including both upgoing and downgoing ray paths. If the velocity contrast between the water layer and the refractor is very large, then the coefficient $\kappa$ becomes unity. In general, however, $\kappa$ is slightly different from unity. Variables $t_{i}, l_{i}$, and $t_{b i}$ are observable variables. In order to take the anisotropic effect in traveltimes into account, we introduce a function of azimuth, $Q(\theta)$, in $v(\theta)$ as

$$
v^{2}(\theta)=\left(v_{0}+\Delta v\right)^{2}+v_{0}^{2} Q(\theta)
$$

with

$$
Q(\theta)=\sum_{j=1}^{N} c_{j} \sin \left(j \theta+\phi_{j}\right),
$$

where $v_{0}+\Delta v$ is an average velocity in the refractor, $c_{j}$ and $\phi_{j}$ are a Fourier coefficient and an initial phase of the $j$-th order, respectively. We have examined the sinusoidal terms up to $\mathrm{N}=6$. For a weakly anisotropic medium $Q(\theta)$ has $2 \theta$ and $4 \theta$ dependencies (Backus, 1965). Under the assumption of

$$
Q(\theta)<1,
$$

Equation (1) can be rewritten as

$$
t_{i}=\left(1-\Delta v / v_{0}\right) l_{i} / v_{0}-Q(\theta) l_{i} /\left(2 v_{0}\right)+t_{0}+\kappa t_{b i}
$$

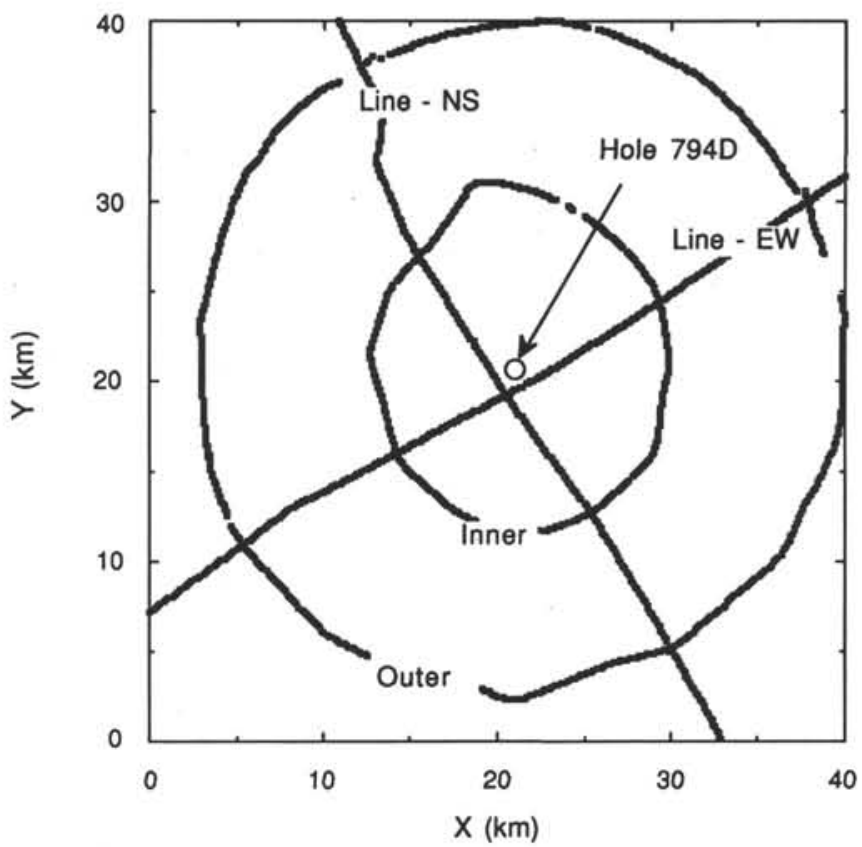

Figure 3. Detailed positions of shots (small dots) showing variation in a shot-receiver distance.

for the weakly anisotropic medium. From $t_{i}, l_{i}, v_{o}$, and $t_{b i}$, we will estimate $t_{0}, \Delta v, \kappa, c_{j}$, and $\phi_{j}$ in a least squares sense.

To examine the statistical significance of introducing the azimuthal dependence in $v(\theta)$, we performed the sequential $F$-test: First, we fitted a model with $Q(\theta)=0$ to our data. Next, we added $c_{1}$, and $\phi_{1}$ in the model $(N=1)$ and fitted it to the data. We performed the $F$-test to see that the introduction of the parameters significantly reduces the sum of squares of residuals. Then, we added $c_{2}$, and $\phi_{2}$ to the model $(N=2)$ to test the significance by calculating the $F$-value of the sum of squares of residuals, and repeated the same procedure until $N$ was 6.

\section{Results}

The result of the F-test for the inner circle shooting observed by the OBDS is listed in Table 1. Our data indicate that introduction of azimuthal dependence is significant up to the sixth-order sinusoidal functions: all anisotropic terms are statistically significant. The sixthorder term is still significant at the 0.01 level. The largest $F$ value (180.8) is obtained for the $2 \theta$ term. The $1 \theta$ term is the next most significant. Observed traveltimes and predicted times by the model up to the sixth for the OBDS are shown in Figure 13. We can see that the data are well fitted by the model. The root mean square of sum of squares for the model without azimuthal dependence $(0.09 \mathrm{~s})$ is reduced to $0.02 \mathrm{~s}$ for the final model. Data from OBSs JRT3, JRT4, and JRT6 are also analyzed and results are tabulated in Tables 2, 3, and 4, respectively. All data indicate significant reduction of residual sum of squares for the $2 \theta$ dependence in the velocity function. Data from the outer circle shooting recorded by the OBDS are in Table 5.

We summarize the final models in Table 6, in which only significant terms were adopted. One standard deviation of estimated parameters is also tabulated in parentheses. The data show that the largest sinusoidal term is the $1 \theta$ for all data. The next largest is $2 \theta$ and then $4 \theta$.

\section{DEVIATION OF PHASE VELOCITY DIRECTION}

Because a water layer is isotropic for $P$-wave propagation, if the underlaying layer is anisotropic, the direction of wave propagation 


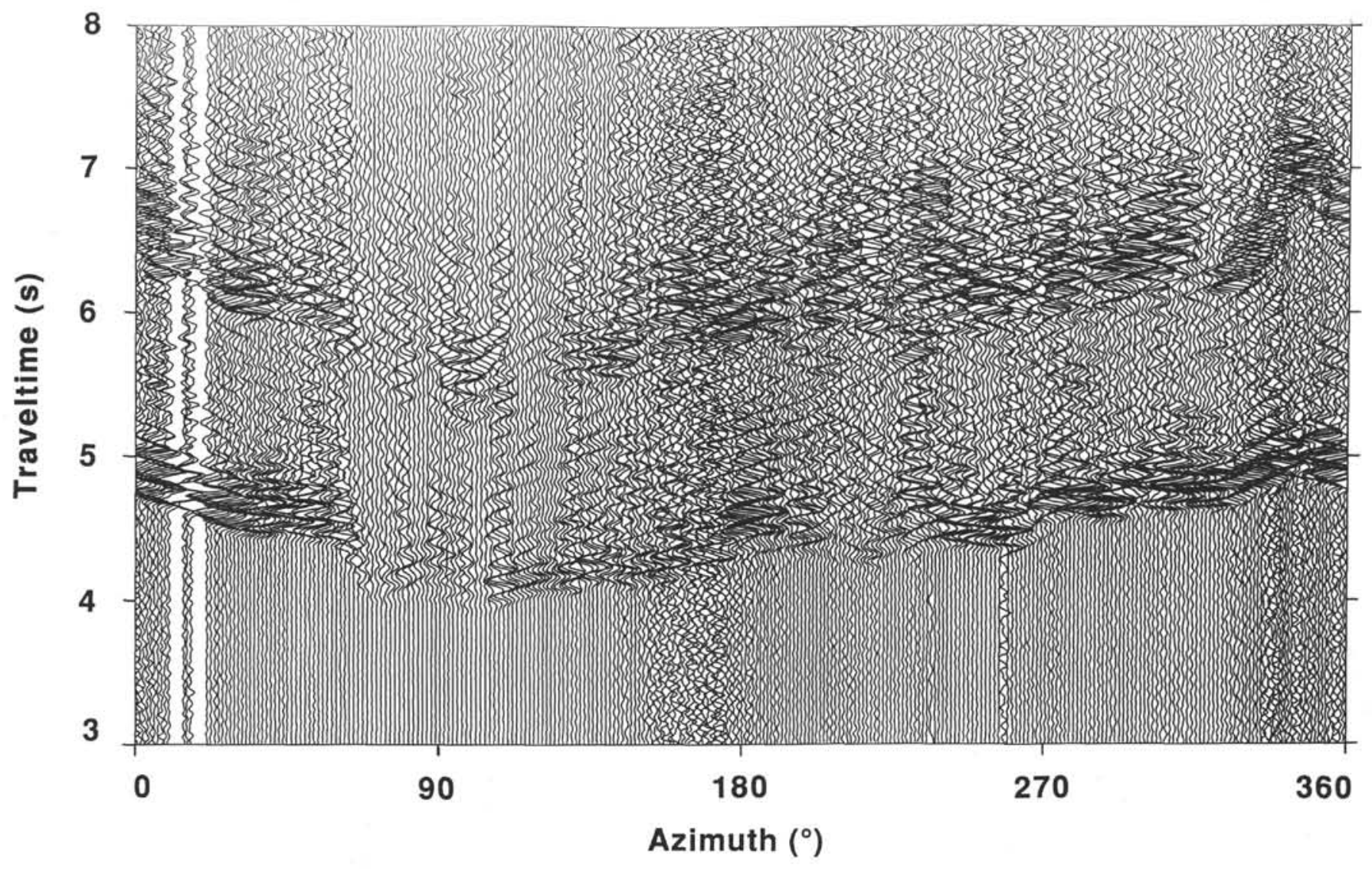

Figure 4. Seismograms of the inner circle shooting observed by the OBDS. The abscissa is azimuth in degrees clockwise from north. The ordinate is traveltime for waves generated by the air gun shot.

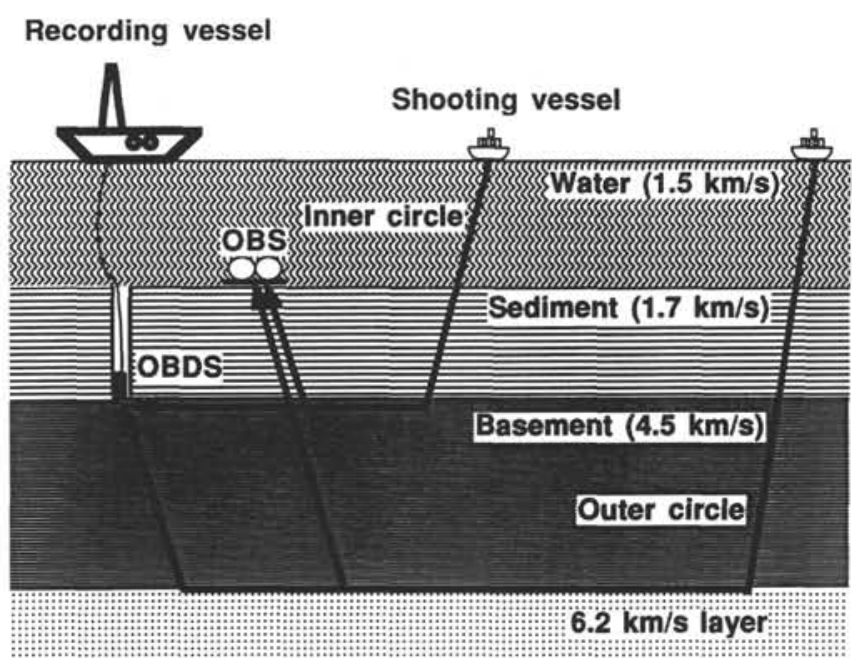

Figure 5. Schematic ray paths and crustal structure near Hole 794D. The crustal structure is derived from air gun profiling data on two linear lines by Shinohara et al. (this volume).

deviates from the sagittal plane on which an incidence wave in the isotropic layer exists (Fig. 14). We estimate the direction of $P$-wave propagation from the OBS array data. The data we used are $\Delta t_{i}^{34}$ and $\Delta t_{i}^{64}$ with $\Delta t_{i}^{34}=t_{i}^{3}-t_{i}^{4}$ and $\Delta t_{i}^{64}=t^{i 6}-t_{i}^{4}$, where $\Delta t_{j} j$ is arrival time observed at $J R T j$ for the $i$-th shot. By using $\Delta t_{i}^{34}$ and $\Delta t_{i}{ }^{64}$, we calculated direction of wave propagation and apparent slowness for each arrival. The direction we measured is normal to the wavefront of the wave which travels across the array: the direction of phase velocity.

Figure 15A shows deviation of the direction from the sagittal plane. It clearly indicates azimuthal dependence of the deviation. The deviation changes its polarity at an azimuth of $180^{\circ}$, which indicates that the east-west direction is the fastest among all directions, suggesting that the array data are consistent with the model with the anisotropic layer. We also calculate the apparent slowness of each arrival (Fig. 15B). The data indicate that waves coming from south are traveling faster than those from the north.

\section{DISCUSSION \\ Inner Circle Shooting}

We have obtained consistent data that suggest anisotropy in the upper crust near Hole 794D. We summarize the fastest direction and the magnitude derived from the $2 \theta$ term in Table 7 . We fitted the $2 \theta$ term to the OBS array data and derived the fastest direction. Traveltime data from the OBDS, OBS JRT4, and JRT6 all indicate that the fastest direction of the $P$-wave propagation is approximately eastsoutheast-west-northwest. We show residuals of traveltimes for the isotropic model $(N=1)$ with respect to azimuth in Figure 16. The residuals can be interpreted as corrected traveltimes for variations of offset distances, depths of the acoustic basement, lateral changes of velocity, and uncontrolled geometrical errors. Figure 16 clearly demonstrates the $2 \theta$ dependence on azimuth which might be masked by lateral heterogeneity in the crust. The statistical test for the inner circle shooting data recorded by JRT4, for example, showed large $F$-values in $N=2$ and 4 , but small or marginal values in $N=3,5$, and 6 (Table 3 ). 

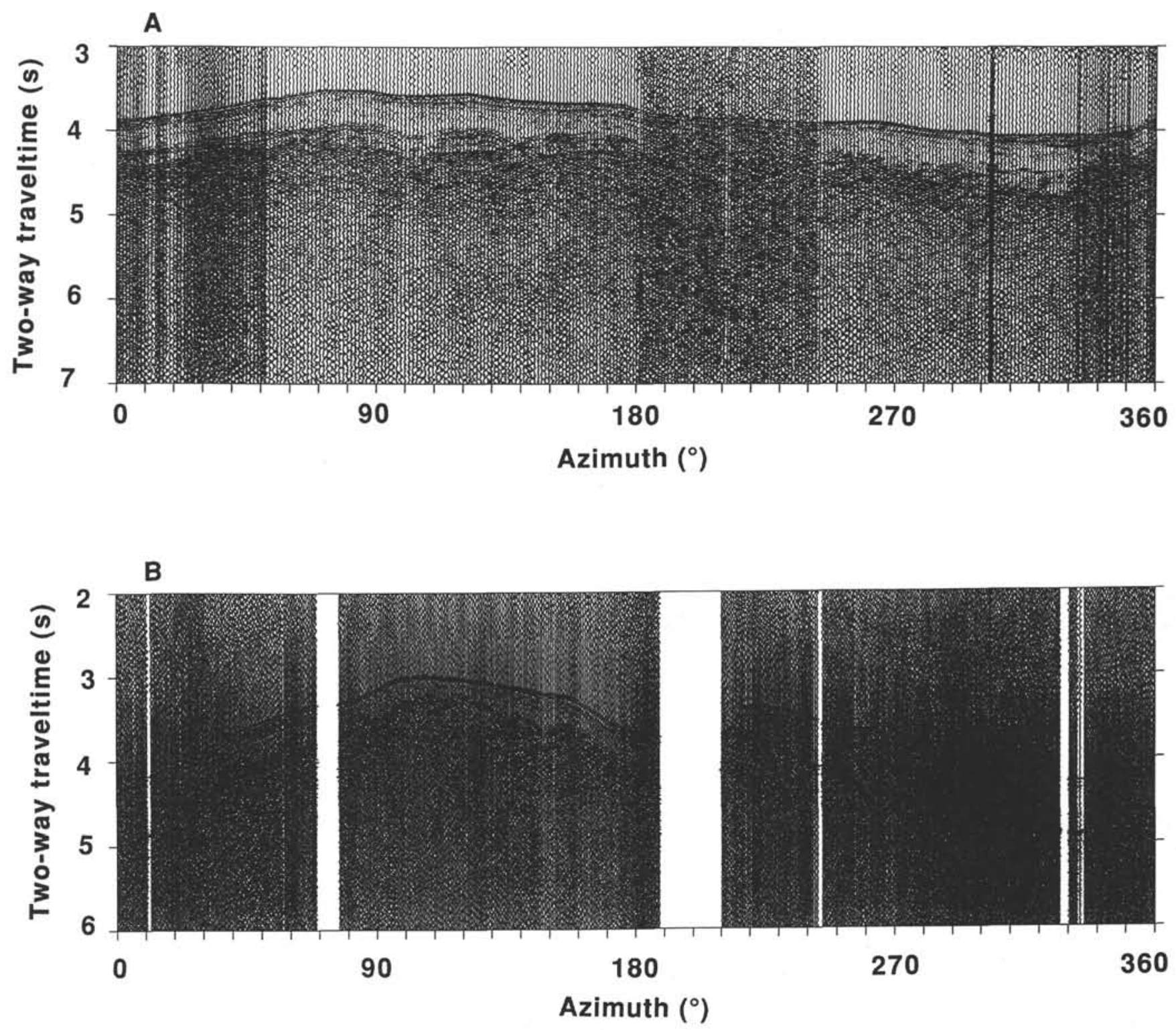

Figure 6. Single-channel reflection profiles showing variation in acoustic basement morphology. The abscissa is azimuth in degrees clockwise from north. A. Inner circle shooting. B. Outer circle shooting.

The result strongly suggests that the effect of anisotropy is dominant in the data. However, there still is a significant component of $1 \theta$. We think that the component is attributed to either lateral change of the velocity in the upper crust or uncorrected error in relative position of the OBS. For example, if the OBS position with respect to the shot points has an error of $100 \mathrm{~m}$, then the effect on the traveltime of the refracted wave for the inner circle shooting is $0.02 \mathrm{~s}$, which is less than $1 \%$ of the traveltime. The amplitude of the $1 \theta$ term $\left(c_{1}\right)$ is $5 \%$, which is larger than the effect due to the error in the position. So we can say that the $1 \theta$ term, together with the higher terms, represents the lateral heterogeneity in the basement layer. Because the amplitudes of the sinusoidal terms are large, the data-set of the OBDS is the most reliable. Table 7 shows that the direction of anisotropy, if any, is scattered in the range from southeast-northwest to east-west. The data from JRT3 for the inner circle shooting has almost northsouth direction of the fastest velocity. The data has the smallest amplitude in $v(\theta)$ and the largest error in estimates. The statistical test for JRT3 also indicates that introduction of the sixth-order term is more significant than that of the second-order term. The reason why the direction of the fastest wave propagation for JRT3 differs from that for OBDS, JRT4, and JRT6 is partly the effect of lateral change of the local structure near JRT3.

\section{Outer Circle Shooting}

The outer circle shooting also indicates azimuthal dependence of propagation in the crust. Since the radius of the outer circle is $18 \mathrm{~km}$, the wave travels down to the $6.2 \mathrm{~km} / \mathrm{s}$ layer (Fig. 5). We can derive information of the deeper crustal structure from the outer circle shooting. Table 7 indicates that the layer has the fastest direction north-south. The direction is almost perpendicular to that derived from the inner circle shooting. One large ambiguity for the outer circle data is, however, variation in the depth of the interface between the $4.5 \mathrm{~km} / \mathrm{s}$ layer and the $6.2 \mathrm{~km} / \mathrm{s}$ layer. The reflection data do constrain the depth of the acoustic basement (Fig. 17), which we used for the analysis of the inner circle data, but do not show the deeper interface. Then, we have two alternative models: an anisotropic and laterally homogeneous model and an isotropic and inhomogeneous model. The 

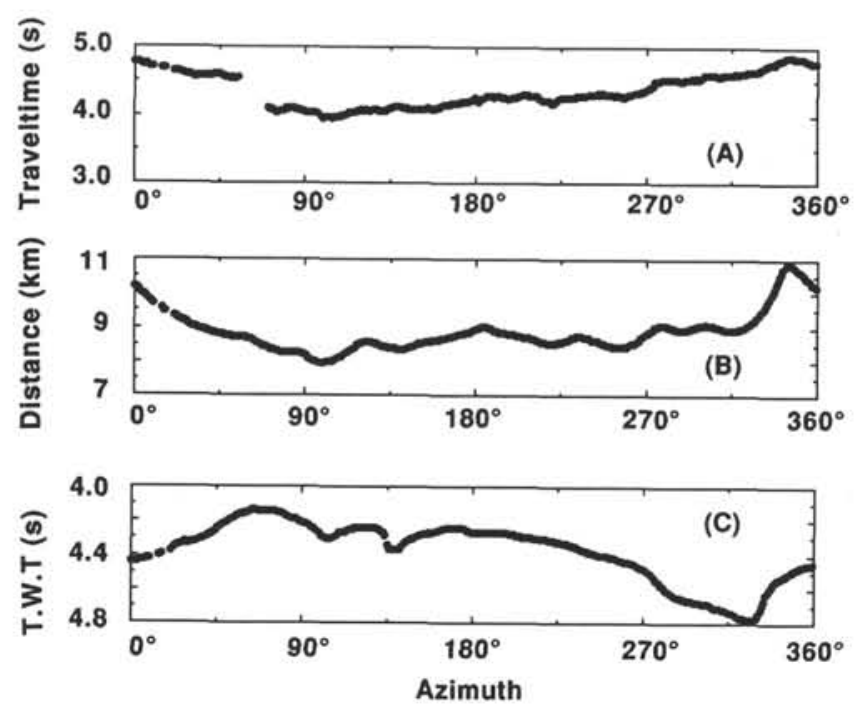

Figure 7. Correlation of traveltimes, offset distances, and depths of the acoustic basement for the inner circle shooting recorded by the OBDS. The abscissa is azimuth in degrees clockwise from north. Traveltime of the refracted wave propagated in the upper crust (top). Offset distance between the shot and the downhole seismometer (center). Two-way traveltime from the sea surface to the acoustic basement (bottom).
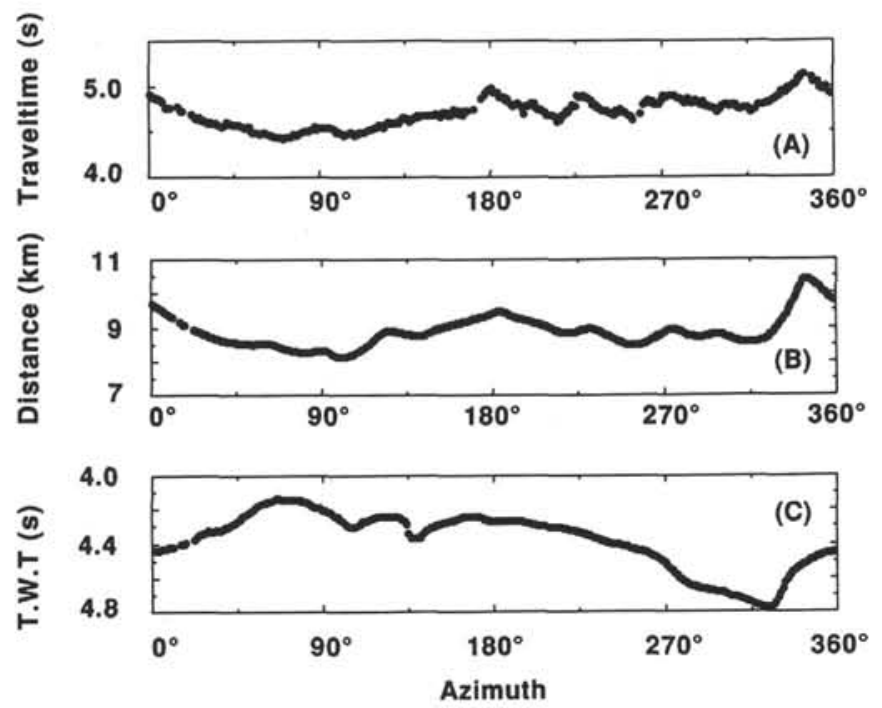

Figure 8. Correlation of traveltimes, offset distances, and depths of the acoustic basement for the inner circle shooting recorded by JRT4. Parameters for plotting are the same as in Figure 7.

latter includes the model in which the thickness of the $4.5 \mathrm{~km} / \mathrm{s}$ layer varies along the outer shooting circle.

It is possible to construct an isotropic model in which the thickness changes: we calculated that contribution from the time term of the $4.5 \mathrm{~km} / \mathrm{s}$ layer, which explains the azimuthal dependence of the traveltime. If the layer has variation in thickness with magnitude of $0.2 \mathrm{~s}$ in time, that is about $900 \mathrm{~m}$, a laterally inhomogeneous model explains the data. We show one of the models in Figure 18: we derived the model by interpolating and extrapolating the variation of the time term on the outer shooting circle into the studied area.
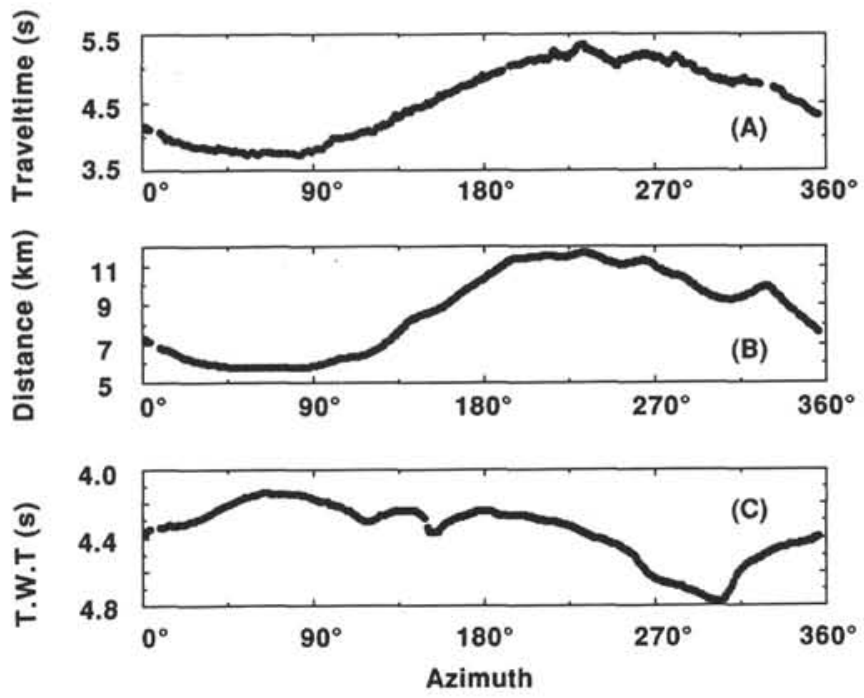

Figure 9. Correlation of traveltimes, offset distances, and depths of the acoustic basement for the inner circle shooting recorded by JRT3. Parameters for plotting are the same as in Figure 7.
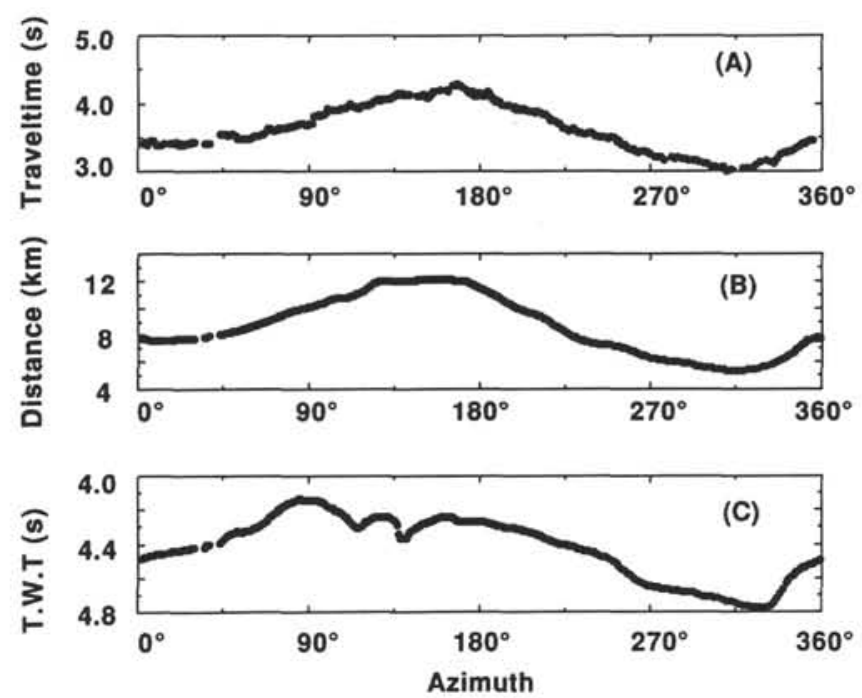

Figure 10. Correlation of traveltimes, offset distances, and depths of the acoustic basement for the inner circle shooting recorded by JRT6. Parameters for plotting are the same as in Figure 7.

\section{Origin of the Anisotropy}

Although the data from the outer circle shooting could not definitely confirm the existence of the anisotropy, we can safely say that the basement layer just below the acoustic basement has a considerable amount of anisotropic component which we detected by analyses of traveltimes and deviation of ray paths (Fig. 19).

We have obtained coring samples from Hole 794D between 573.0 and 733.5 mbsf (Ingle, Suyehiro, von Breymann, et al., 1990). Within the depth range we found nine igneous units: the lithologic sequence represents a series of stacked sills and flows. The igneous complex represents a portion of the Miocene volcanic floor. A $P$-wave velocity analyzed in samples from the sequence shows a minimum of $3.8 \mathrm{~km} / \mathrm{s}$ 


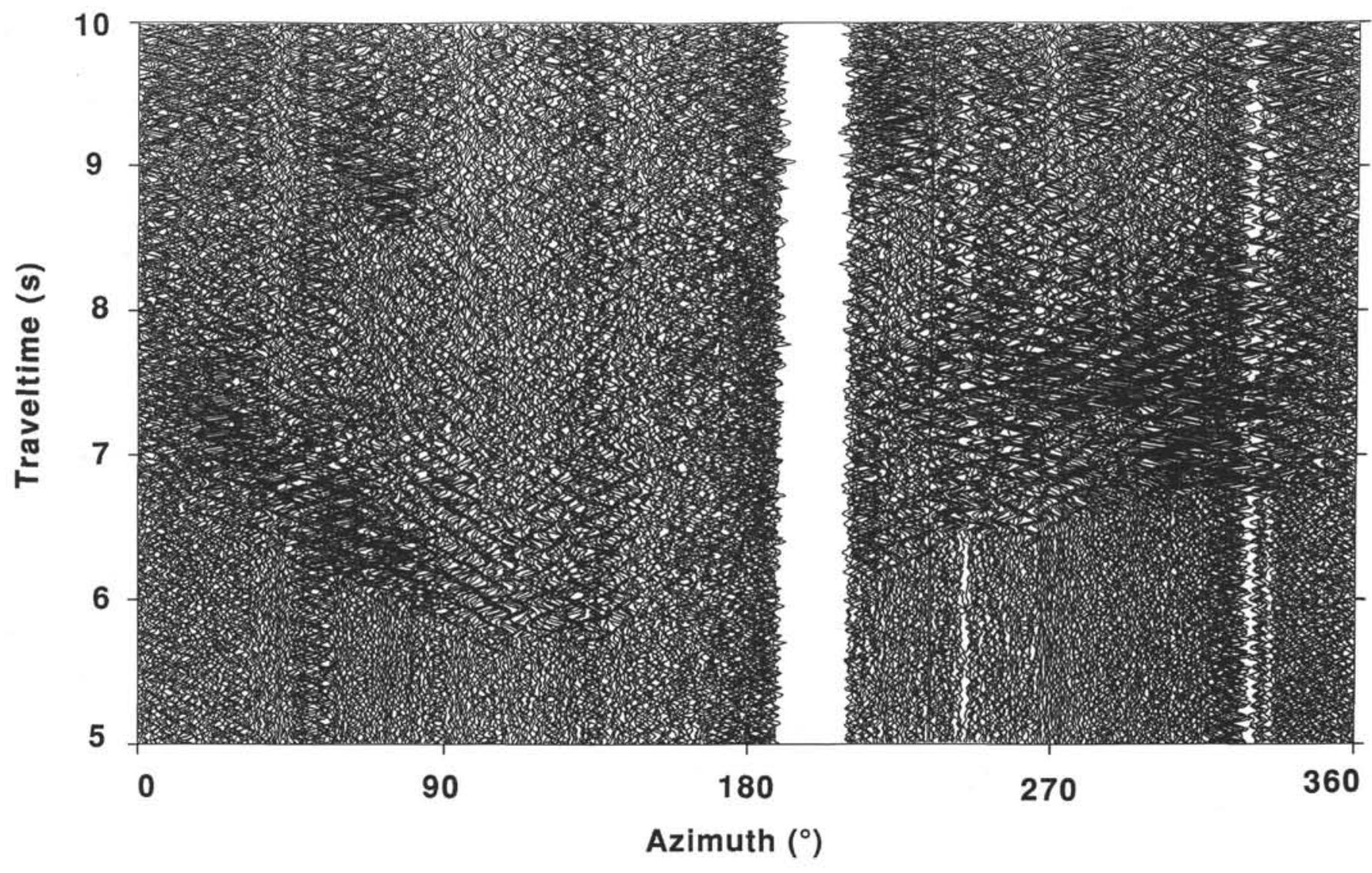

Figure 11. Seismograms of the outer circle shooting observed by the OBDS. The abscissa is azimuth in degrees clockwise from north. The ordinate is traveltime for waves generated by the air gun shot.
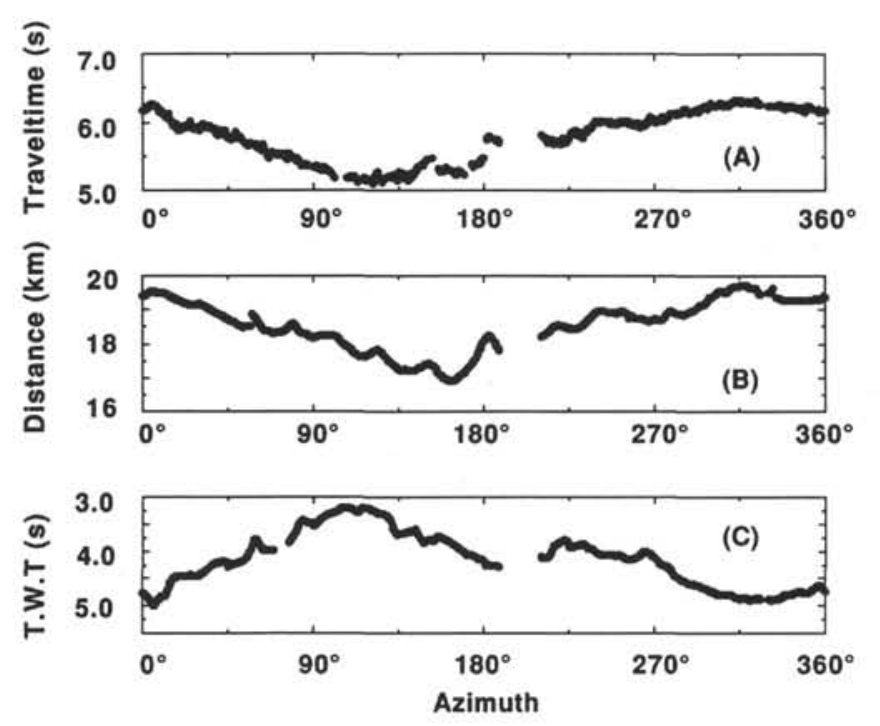

Figure 12. Correlation of traveltimes, offset distances, and depths of the acoustic basement for the outer circle shooting recorded by the downhole seismometer. The abscissa is azimuth in degrees clockwise from north. Traveltime of the refracted wave propagating in the upper $6.2 \mathrm{~km} / \mathrm{s}$ layer (top). Offset distance between the shot and the OBDS in km (center). Two-way traveltime from the sea surface to the acoustic basement (bottom).
Table 1. Analysis of variance for data from the OBDS for the inner circle shooting.

\begin{tabular}{ccccc}
\hline N & df & SS $\left(\mathrm{s}^{2}\right)$ & RMS $(s)$ & F \\
\hline 0 & 265 & 2.065 & 0.088 & - \\
1 & 263 & 0.997 & 0.062 & 140.86 \\
2 & 261 & 0.418 & 0.040 & 180.76 \\
3 & 259 & 0.263 & 0.032 & 76.32 \\
4 & 257 & 0.177 & 0.026 & 62.43 \\
5 & 255 & 0.158 & 0.025 & 15.33 \\
6 & 253 & 0.128 & 0.022 & 29.65 \\
\hline
\end{tabular}

df = degree of freedom; SS = sum of squares of residuals; RMS = root mean square of sum of residuals; $\mathrm{F}=$ calculated sequential $\mathrm{F}$. Critical point of $\mathrm{F}(2, \infty, 0.01)$ is 4.61 .

at $699 \mathrm{mbsf}$ and a maximum of $6.3 \mathrm{~km} / \mathrm{s}$ at $667 \mathrm{mbsf}$. Moreover, since the core recovery is $21.7 \%$ at the hole, we lost many soft materials in the sequence. These observations suggest highly heterogeneous structure in that depth range.

A small-scale heterogeneity whose characteristic length is less than the wavelength of the seismic wave can cause an anisotropic effect on wave propagation. Since the wavelength of the $P$-wave in the basement layer is a few hundred meters, there should appear an anisotropic effect if the heterogeneities of a scale of about several tens of meters are aligned. Sills or dikes in the upper crust may account for such heterogeneity. The direction of the strike of intrusion of the 


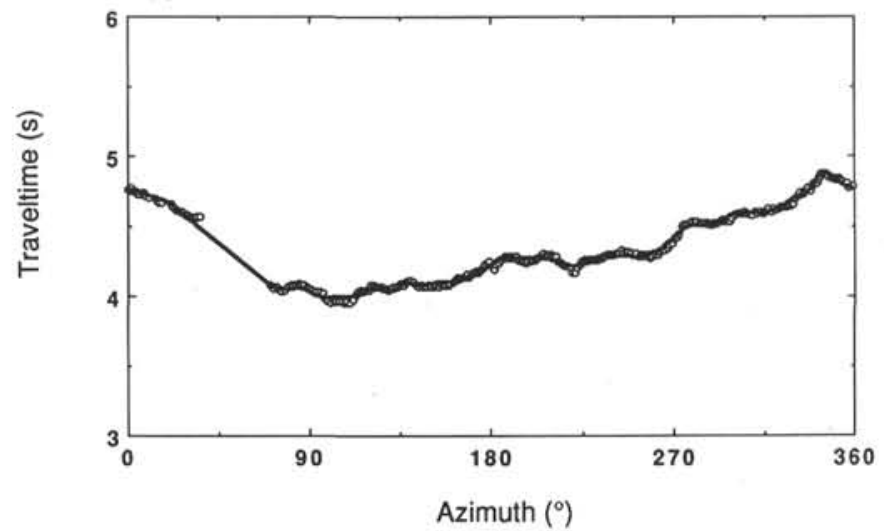

C

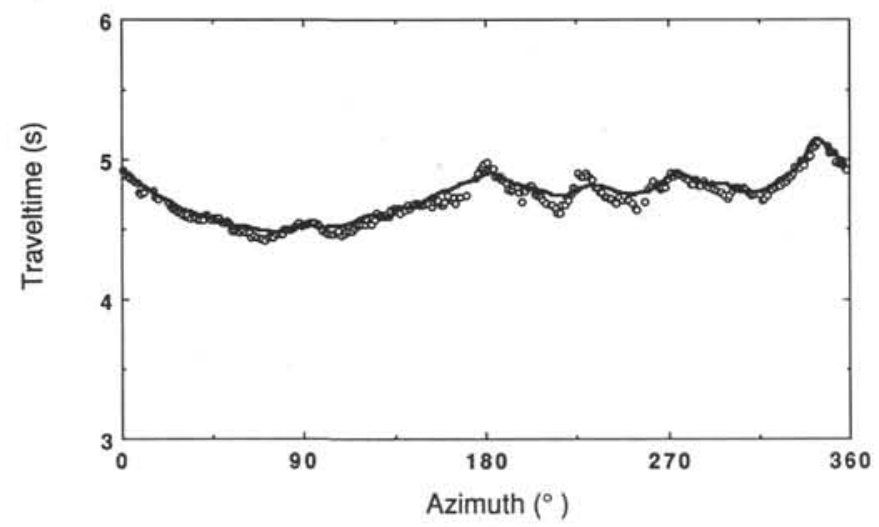

B

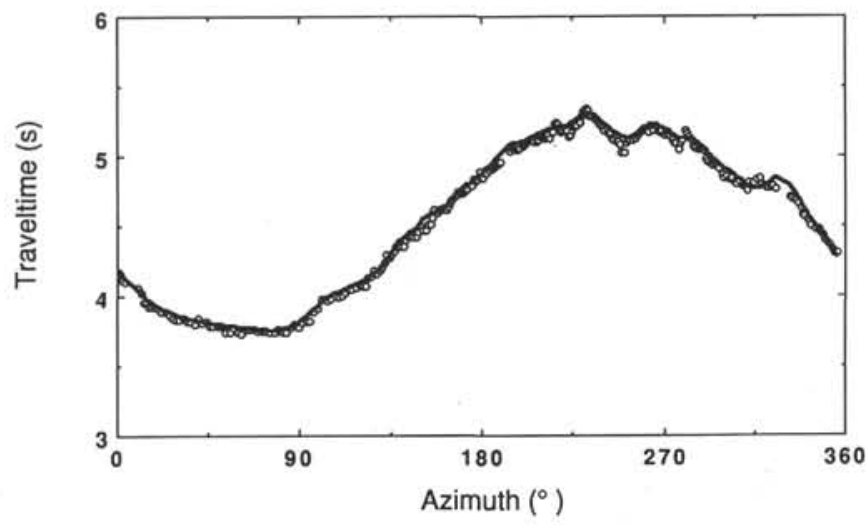

D

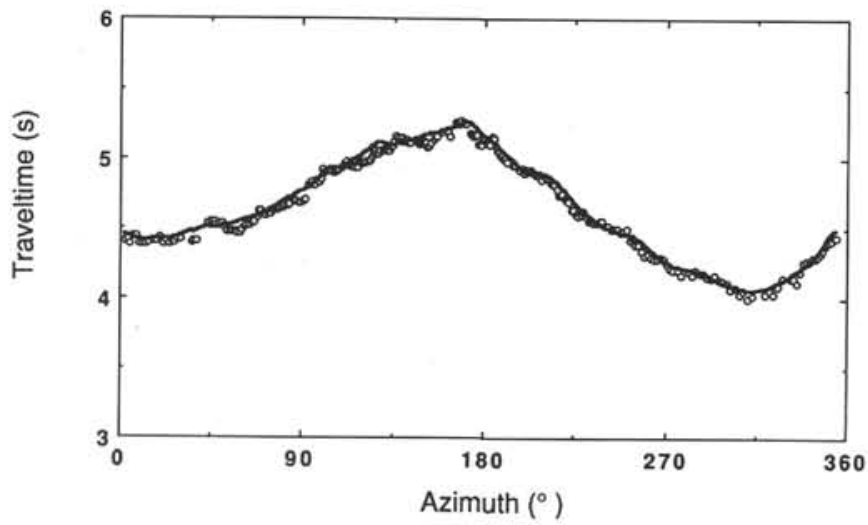

E

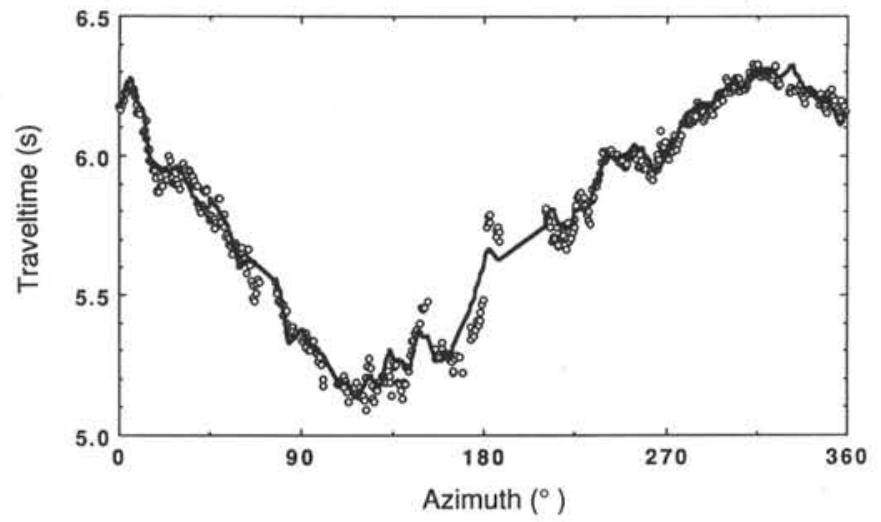

Figure 13. Observed traveltimes for the refraction arrivals (circles) and calculated traveltimes by the best fitted models in Table 6 (solid lines). A. Inner circle shooting recorded by the OBDS. B. JRT3. C. JRT4. D. JRT6. E. Outer circle shooting by the OBDS.

dike is controlled by the stress field during the igneous activity: the strike is parallel to the direction of the maximum principal axis of the tectonic stress (e.g., Nakamura, 1977). The alignment of the intrusion is parallel to the fastest direction of the wave propagation because the igneous material is generally hard and has a higher wave velocity. Thus, if this mechanism was dominant, our data suggest that there was the east-west to east-southeast-west-northwest compressional stress field at the formation of the crust. This inference contradicts the scenario that the Japan Sea was opened due to a back-arc spreading at an age of about 15-20 Ma (e.g., Otofuji et al., 1985) if the small-scale local structure is also controlled by the tensional stress field of east-southeast-west-northwest direction in the regional scale.

We therefore prefer the idea that the present stress field makes the preferred orientation of the heterogeneity in the upper crust: earthquake focal mechanisms indicate that the stress state is compressional in the east-west to east-southeast-west-northwest direction (Fig. 20; Yamazaki et al., 1985). If a medium with randomly oriented cracks is deformed due to a compressional stress field, cracks perpendicular to 
Table 2. Analysis of variance for data from JRT3 for the inner circle shooting.

\begin{tabular}{ccccc}
\hline N & df & SS $\left(\mathrm{s}^{2}\right)$ & RMS (s) & \multicolumn{1}{c}{ F } \\
\hline 0 & 280 & 0.694 & 0.050 & - \\
1 & 278 & 0.471 & 0.041 & 68.17 \\
2 & 276 & 0.402 & 0.038 & 24.66 \\
3 & 274 & 0.322 & 0.034 & 35.46 \\
4 & 272 & 0.307 & 0.034 & 6.75 \\
5 & 270 & 0.299 & 0.033 & 3.55 \\
6 & 270 & 0.218 & 0.028 & 51.95 \\
\hline
\end{tabular}

$\mathrm{df}=$ degree of freedom; $\mathrm{SS}=$ sum of squares of residuals; $\mathrm{RMS}=$ root mean square of sum of residuals; $F=$ calculated sequential $F$. Critical point of $F(2, \infty, 0.01)$ is 4.61 .

Table 3. Analysis of variance for data from JRT4 for the inner circle shooting.

\begin{tabular}{ccccr}
\hline $\mathrm{N}$ & df & SS $\left(\mathrm{s}^{2}\right)$ & RMS $(\mathrm{s})$ & \multicolumn{1}{c}{$\mathrm{F}$} \\
\hline 0 & 278 & 1.130 & 0.064 & - \\
1 & 276 & 0.779 & 0.053 & 62.18 \\
2 & 274 & 0.599 & 0.047 & 41.20 \\
3 & 272 & 0.597 & 0.047 & 0.46 \\
4 & 272 & 0.453 & 0.041 & 43.20 \\
5 & 270 & 0.435 & 0.040 & 5.65 \\
6 & 268 & 0.422 & 0.040 & 4.16 \\
\hline
\end{tabular}

$\mathrm{df}=$ degree of freedom; $\mathrm{SS}=$ sum of squares of residuals; $\mathrm{RMS}=$ root mean square of sum of residuals; $\mathrm{F}=$ calculated sequential $\mathrm{F}$. Critical point of $\mathrm{F}(2, \infty, 0.01)$ is 4.61 .

Table 4. Analysis of variance for data from JRT6 for the inner circle shooting.

\begin{tabular}{ccccr}
\hline $\mathrm{N}$ & df & SS $\left(\mathrm{s}^{2}\right)$ & RMS $(\mathrm{s})$ & \multicolumn{1}{c}{$\mathrm{F}$} \\
\hline 0 & 265 & 2.860 & 0.104 & - \\
1 & 263 & 1.589 & 0.078 & 105.18 \\
2 & 261 & 0.577 & 0.047 & 229.00 \\
3 & 259 & 0.393 & 0.039 & 60.82 \\
4 & 257 & 0.390 & 0.039 & 0.96 \\
5 & 257 & 0.346 & 0.037 & 17.48 \\
6 & 255 & 0.273 & 0.033 & 33.73 \\
\hline
\end{tabular}

$\mathrm{df}=$ degree of freedom; $\mathrm{SS}=$ sum of squares of residuals; RMS = root mean square of sum of residuals; $F=$ calculated sequential $F$. Critical point of $F(2, \infty, 0.01)$ is 4.61 .

Table 5. Analysis of variance for data from the OBDS for the outer circle shooting.

\begin{tabular}{ccccc}
\hline $\mathrm{N}$ & df & SS $\left(\mathrm{s}^{2}\right)$ & RMS $(\mathrm{s})$ & $\mathrm{F}$ \\
\hline 0 & 522 & 6.674 & 0.113 & - \\
1 & 520 & 3.315 & 0.080 & 263.42 \\
2 & 518 & 1.900 & 0.061 & 193.12 \\
3 & 516 & 1.538 & 0.055 & 60.56 \\
4 & 514 & 1.455 & 0.053 & 14.70 \\
5 & 512 & 1.372 & 0.052 & 15.56 \\
6 & 510 & 1.217 & 0.049 & 32.34 \\
\hline
\end{tabular}

df = degree of freedom; $S S$ = sum of squares of residuals; RMS = root mean square of sum of residuals; $F=$ calculated sequential $F$. Critical point of $F(2, \infty, 0.01)$ is 4.61 .

the maximum stress direction close and those parallel to the direction open (Fig. 21). Eventually, the open cracks align in the direction of the compressional stress axis, and a macroscopic velocity is also fastest in the direction. Our observation of the anisotropy is consistent with this idea. The cracks, in reality, can be some soft materials in the igneous rocks of the upper crust. Our data also suggest strong lateral heterogeneity in the upper crust: the structure with a scale length greater than the wavelength appears as heterogeneity.
There still remains work in the future, which is beyond the scope of the present study: we should treat both anisotropic and lateral inhomogeneous effects simultaneously in the traveltime analysis. Then we will be able to discuss the magnitude of anisotropy and heterogeneity.

\section{CONCLUSIONS}

We conducted a controlled source seismic experiment to detect seismic anisotropy in the upper crust using data from the OBDS installed in Hole 794D of the Ocean Drilling Program. Combining data from OBSs, which were deployed during the experiment, we obtained refraction and reflection data on two circle profiles with radii of 9 and $19 \mathrm{~km}$. Data from the inner circle shooting provided confident evidences of the anisotropy and the lateral heterogeneity: we estimated the direction and the magnitude of the anisotropy by modeling the traveltime data with sinusoidal terms in a velocity function of the refracted waves. The fastest direction is east-west to east-southeastwest-northwest and the magnitude is in the range of $3 \%$ to $7 \%$. We interpret the anisotropy to have been caused by the present stress field of the east-west to southeast-northwest compression.

\section{ACKNOWLEDGMENTS}

We are grateful to Prof. T. Kanazawa for his help in operation of the ocean broadband downhole seismometer. We owe very much to ODP engineers and technicians and the Schlumberger engineer aboard the JOIDES Resolution during Leg 128 . We thank all the onboard scientists including Prof. H. Kinoshita, Drs. H. Shiobara, and A. Nishizawa aboard the Tansei-maru, University of Tokyo.

\section{REFERENCES}

Backus, G. E., 1965. Possible forms of seismic anisotropy of the upper-most mantle under oceans. J. Geophys. Res., 70:3429-3439.

Chung, T. W., Hirata, N., and Sato, R., 1990. Two-dimensional P-and S-wave velocity structure of the Yamato Basin, the southeastern Japan Sea, from refraction data collected by an ocean bottom seismographic array. J. Phys. Earth, 38:99-147.

1992. A quantitative study of seismic anisotropy in the Yamato Basin, the southeastern Japan Sea, from refraction data collected by an ocean bottom seismographic array. Geophys. J. Int.

Hirata, N., Kinoshita, H., Suyehiro, K., Suyemasu, M., Matsuda, N.,Ouchi, T., Katao, H., Koresawa, S., and Nagumo, S., 1987. Report on DELP 1985 cruises in the Japan Sea. Part II: seismic refraction experiment conducted in the Yamato Basin, southeast Japan Sea. Tokyo Daigaku Jishin Kenkyusho Iho, 62:347-365.

Hirata, N., Tokuyama, H., and Chung, T. W., 1989. An anomalously thick layering of the crust of the Yamato Basin, southeastern Sea of Japan: the final stage of back-arc spreading. Tectonophysics, 165:303-314.

Ingle, J. C., Jr., Suyehiro, K., von Breymann, M. T., et al., 1990. Proc. ODP. Init. Repts., 128: College Station, TX (Ocean Drilling Program).

Katao, H., 1988. Seismic structure and formation of the Yamato Basin. Tokyo Daigaku Jishin Kenkyusho Tho, 63:51-86.

Little, S. A., and Stephen, R. A., 1985. Costa Rica rift borehole seismic experiment, Deep Sea Drilling Project Hole 504B, Leg 92. In Anderson, R. N., Honnorez, J., Becker, K., et al., Init. Repts. DSDP, 83: Washington (U.S. Govt. Printing Office), 517-528.

Ludwig, W. J., Murauchi, S., and Houtz, R. E., 1975. Sediments and structure of the Japan Sea. Geol. Soc. Am. Bull., 86:651-664.

Murauchi, S., 1972. Crustal structure of the Japan Sea. Kagaku, 42:367-375. (in Japanese)

Nakamura, K., 1977. Volcanoes as possible indicators of tectonic stress orientation-principle and proposal. J. Volcanol. Geotherm. Res., 2:1-16.

Okada, H., Moriya, T., Masuda, T., Hasegawa, T., Asano, S., Kasahara, K., Ikami, A., Aoki, H., Sasaki, Y., Hurukawa, N., and Matsumura, K., 1978. Velocity anisotropy in the Sea of Japan as revealed by big explosions. $J$. Phys. Earth, 26:491-502.

Otofuji, Y., Hayashida, A., and Torii, M., 1985. When was the Japan Sea opened?: paleomagnetic evidence from southwest Japan. In Nasu, N., Kushiro, I., Kobayashi, K., and Kagami, H. (Eds.), Formation of Active Ocean Margins: Tokyo (Terrapub), 551-566. 
Shearer, P., and Orcutt, J., 1985. Anisotropy in the oceanic lithosphere - theory and observations from the Ngendei seismic refraction experiment in the south-west Pacific. Geophys. J. R. Astron. Soc., 80:493-526.

Stephen, R. A., 1981. Seismic anisotropy observed in upper oceanic crust. Geophys. Res. Lett., 8:865-868.

1985. Seismic anisotropy in the upper oceanic crust. J. Geophys. Res., 90:11383-11396.

, 1988. Lateral heterogeneity in the upper oceanic crust at Deep Ocean Drilling Project Site 504. J. Geophys. Res., 93:6571-6584.

White, R. S., and Whitmarsh, R. B., 1984. An investigation of seismic anisotropy due to cracks in the upper oceanic crust at $45^{\circ} \mathrm{N}$, mid-Atlantic ridge. Geophys. J. R. Astron. Soc., 79:439-467.
Yamazaki, K., Tamura, T., and Kawasaki, I., 1985. Seismogenic stress field of the Japan Sea as derived from shallow and small earthquakes. Zisin (J. Seismol. Soc. Jpn.), 38:541-558. (in Japanese with English abstract)

Date of initial receipt: 26 March 1991

Date of acceptance: 7 August 1991

Ms 127/128B-231 
Table 6. Anisotropic models.

\begin{tabular}{llllcccc}
\hline Seismometer & $\begin{array}{c}t_{0} \\
(\mathrm{~s})\end{array}$ & $\begin{array}{l}\Delta v / v_{0} \\
(\%)\end{array}$ & $\kappa$ & $\begin{array}{c}c_{1} \\
(\%)\end{array}$ & $\begin{array}{c}\phi_{1} \\
\left({ }^{\circ}\right)\end{array}$ & $\begin{array}{c}c_{2} \\
(\%)\end{array}$ & $\begin{array}{c}\phi_{2} \\
\left({ }^{\circ}\right)\end{array}$ \\
\hline OBDS (inner) & - & 3.900 & 1.043 & 9.750 & -66.34 & 6.610 & -157.15 \\
& - & $(0.076)$ & $(0.070)$ & $(0.085)$ & $(0.77)$ & $(0.115)$ & $(0.91)$ \\
JRT3 & 0.299 & 0.376 & 1.054 & 5.529 & 99.85 & 3.196 & 59.72 \\
& $(0.005)$ & $(0.096)$ & $(0.009)$ & $(0.994)$ & $(12.20)$ & $(1.089)$ & $(18.71)$ \\
JRT4 & 0.300 & 0.235 & 1.112 & 5.189 & 67.61 & 3.801 & 162.71 \\
& $(0.005)$ & $(0.096)$ & $(0.009)$ & $(0.844)$ & $(9.67)$ & $(0.859)$ & $(13.07)$ \\
JRT6 & 0.300 & 0.130 & 1.085 & 7.466 & 333.82 & 7.302 & 167.60 \\
& $(0.005)$ & $(0.096)$ & $(0.009)$ & $(1.195)$ & $(8.42)$ & $(1.130)$ & $(8.19)$ \\
OBDS (outer) & 0.747 & 0.158 & 0.988 & 6.630 & 4.44 & 5.309 & 110.10 \\
& $(0.001)$ & $(0.010)$ & $(0.001)$ & $(0.043)$ & $(0.41)$ & $(0.044)$ & $(0.49)$ \\
\hline
\end{tabular}

Table 6 (continued).

\begin{tabular}{lcccccccc}
\hline Seismometer & $c 3$ & $\phi 3$ & $c 4$ & $\phi 4$ & $c 5$ & $\phi 5$ & $c 6$ & $\begin{array}{c}\phi 6 \\
(\%) \\
\end{array}$ \\
& $(\%)$ & $\left({ }^{\circ}\right)$ & $(\%)$ & $\left({ }^{\circ}\right)$ & $(\%)$ & $\left({ }^{\circ}\right)$ & $(\%)$ & $\left(\begin{array}{l}{ }^{\circ} \\
(\%)\end{array}\right.$ \\
\hline OBDS (inner) & 2.180 & 160.75 & 2.730 & 172.55 & 1.700 & 139.85 & 2.390 & 140.2 \\
& $(0.104)$ & $(2.96)$ & $(0.096)$ & $(2.38)$ & $(0.104)$ & $(3.33)$ & $(0.105)$ & $(2.17)$ \\
JRT3 & 2.151 & -54.07 & 1.018 & 116.96 & - & - & 2.380 & -22.75 \\
& $(1.072)$ & $(27.54)$ & $(0.913)$ & $(53.37)$ & - & - & $(0.814)$ & $(19.49)$ \\
JRT4 & - & - & 3.997 & 198.90 & 0.709 & 248.64 & - & - \\
& - & - & $(0.847)$ & $(12.49)$ & $(0.857)$ & $(69.40)$ & - & - \\
JRT6 & 2.719 & 350.52 & - & - & 1.397 & 124.01 & 1.841 & 351.10 \\
& $(0.979)$ & $(19.98)$ & - & - & $(0.928)$ & $(39.83)$ & $(0.936)$ & $(29.64)$ \\
OBDS (outer) & 2.659 & 255.90 & 1.071 & 22.42 & 0.960 & 347.50 & 1.583 & 16.11 \\
& $(0.044)$ & $(0.97)$ & $(0.048)$ & $(2.19)$ & $(0.046)$ & $(2.56)$ & $(0.044)$ & $(1.53)$ \\
\hline
\end{tabular}

One standard deviation of the estimates in parenthesis. 


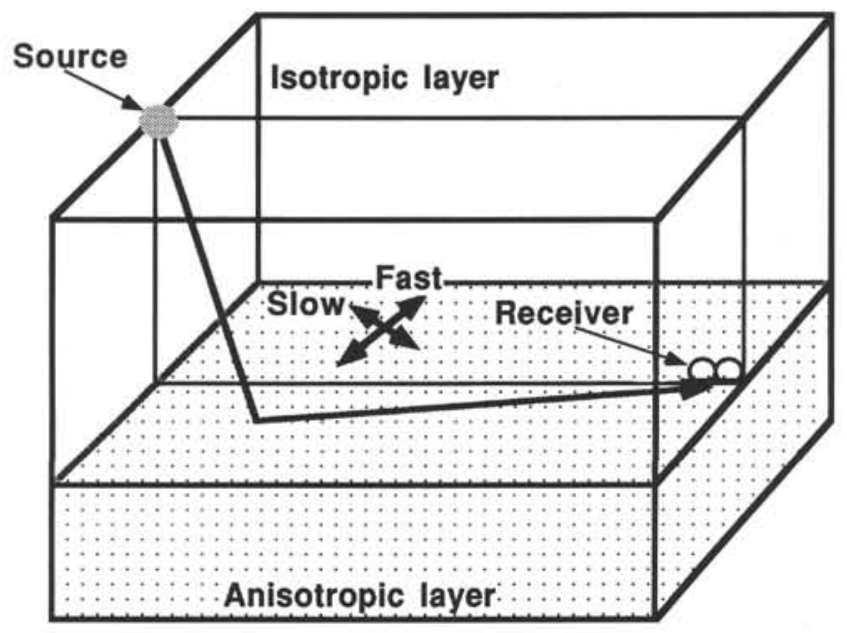

Figure 14. Schematic ray diagram for an isotropic medium underlain by an anisotropic medium.
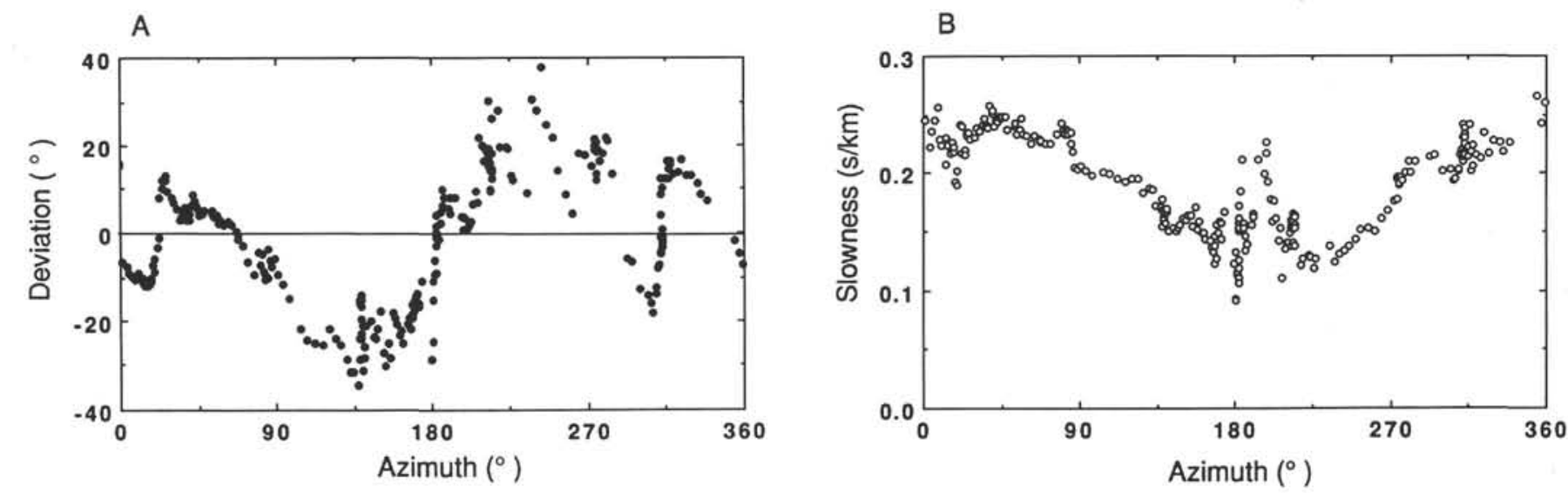

Figure 15. Slowness vectors determined from the OBS array data. A. Deviation of direction of phase velocity from the source-receiver azimuth in degrees, positive for clockwise. B. Magnitude of slowness in $\mathrm{s} / \mathrm{km}$.

Table 7. Direction and magnitude of anisotropy.

\begin{tabular}{ccc}
\hline DATA & $\begin{array}{c}\text { Fastest direction } \\
\left({ }^{\circ}\right)\end{array}$ & $\begin{array}{c}\text { Magnitude } \\
(\%)\end{array}$ \\
\hline OBDS (inner) & $123.58 \pm 0.45$ & $6.61 \pm 0.12$ \\
JRT3 & $15.14 \pm 9.35$ & $3.20 \pm 1.09$ \\
JRT4 & $143.95 \pm 6.54$ & $3.80 \pm 0.86$ \\
JRT6 & $141.20 \pm 4.10$ & $7.30 \pm 1.13$ \\
OBDS (outer) & $169.95 \pm 0.25$ & $5.31 \pm 0.04$ \\
OBSs array & 93.50 & - \\
\hline
\end{tabular}

Magnitude $=\left(V_{\max }-V_{\min }\right) / V_{0}$. Error in the estimate is shown by one standard error. 

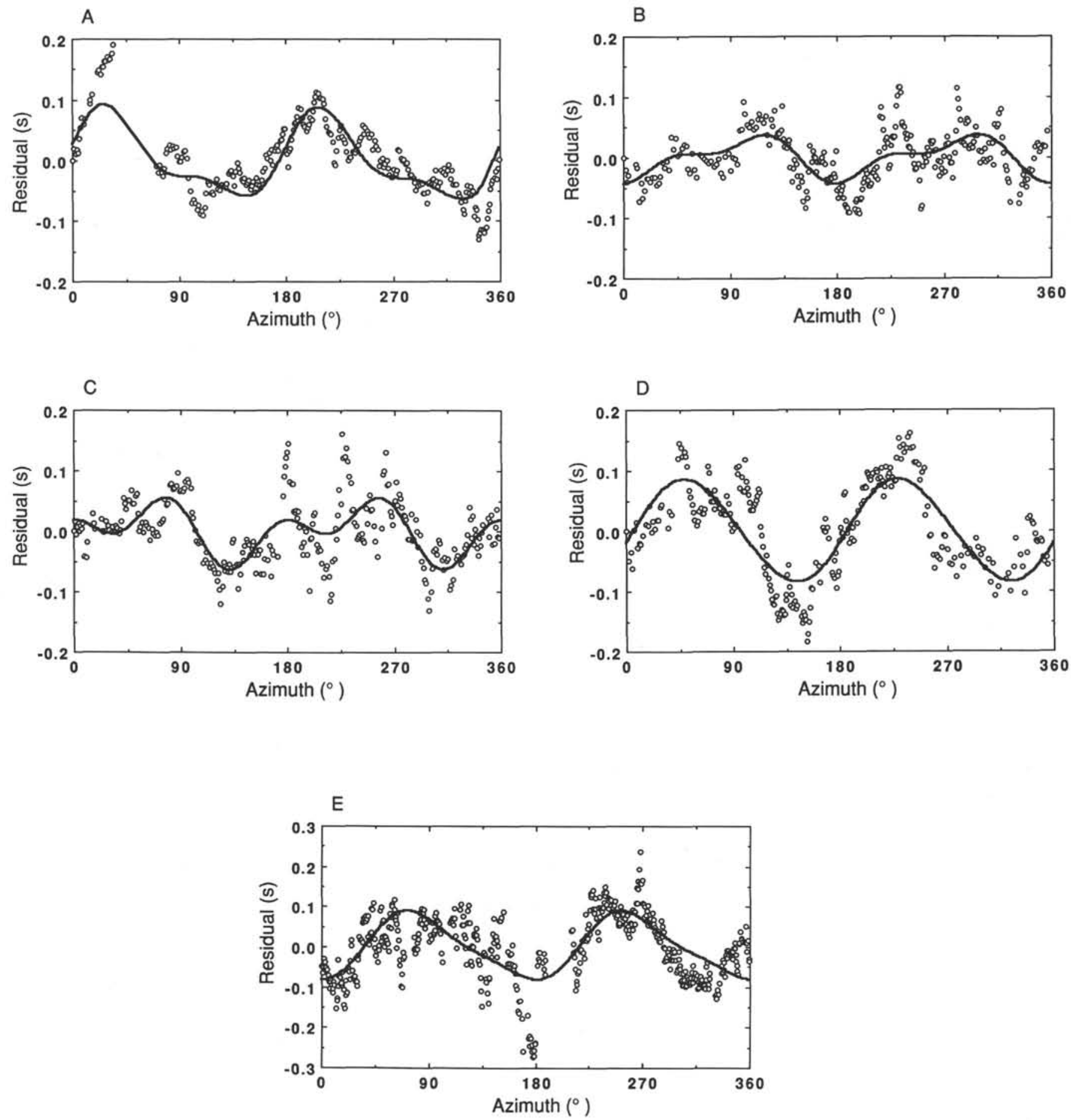

Figure 16. Residuals of traveltimes for the isotropic models $(\mathrm{N}=1)$. Circles indicate traveltime residuals. A line shows the model with isotropic, $2 \theta$ and $4 \theta$ terms. A. Inner circle shooting recorded by the OBDS. B. JRT3. C. JRT4. D. JRT6. E. Outer circle shooting by the OBDS. 


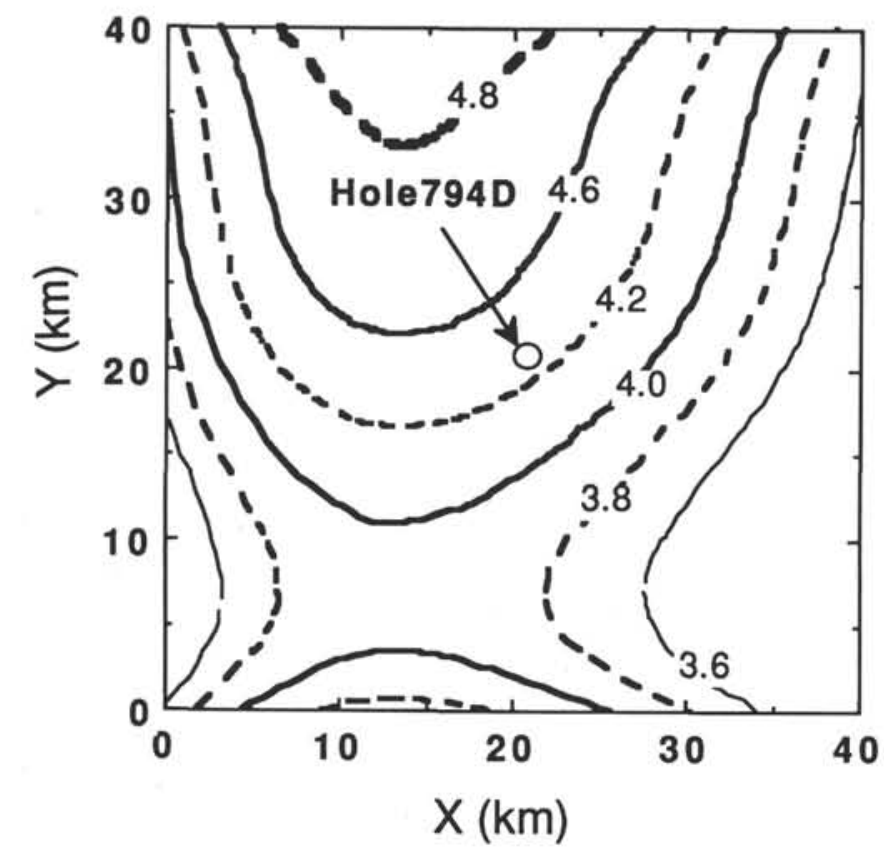

Figure 17. Depth of the acoustic basement. Contours show two-way traveltimes from sea surface in $\mathrm{s}$.

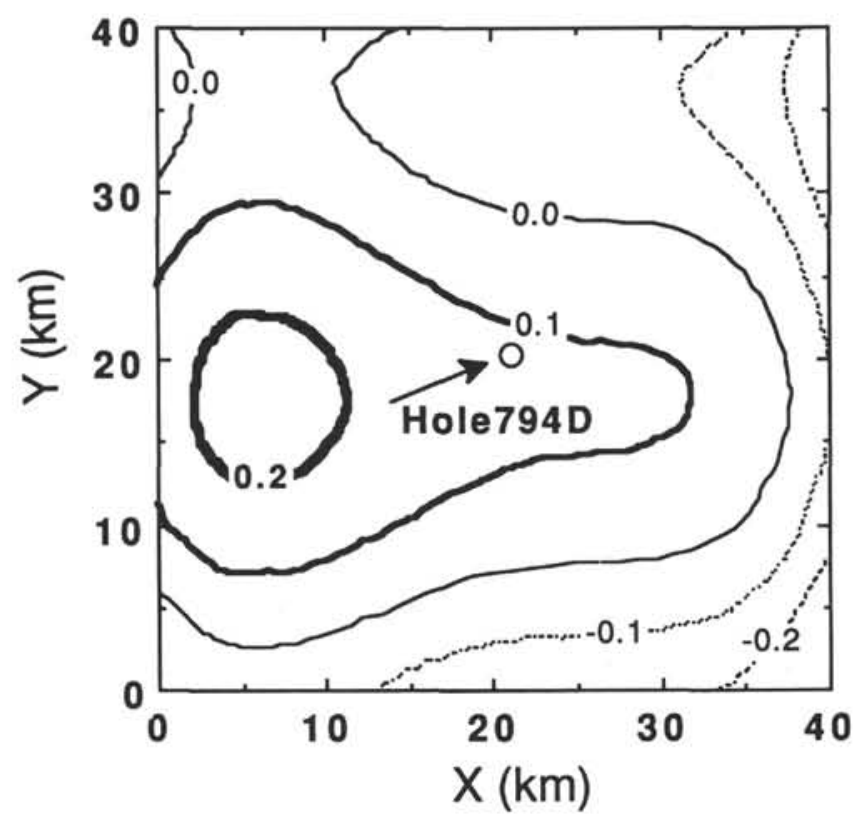

Figure 18. Variation of thickness of the $4.5 \mathrm{~km} / \mathrm{s}$ layer. Assuming that the variation in the traveltime of the refracted wave from the outer circle shooting is due to the variation of the thickness, then the contribution to the time is shown in $\mathrm{s}$.

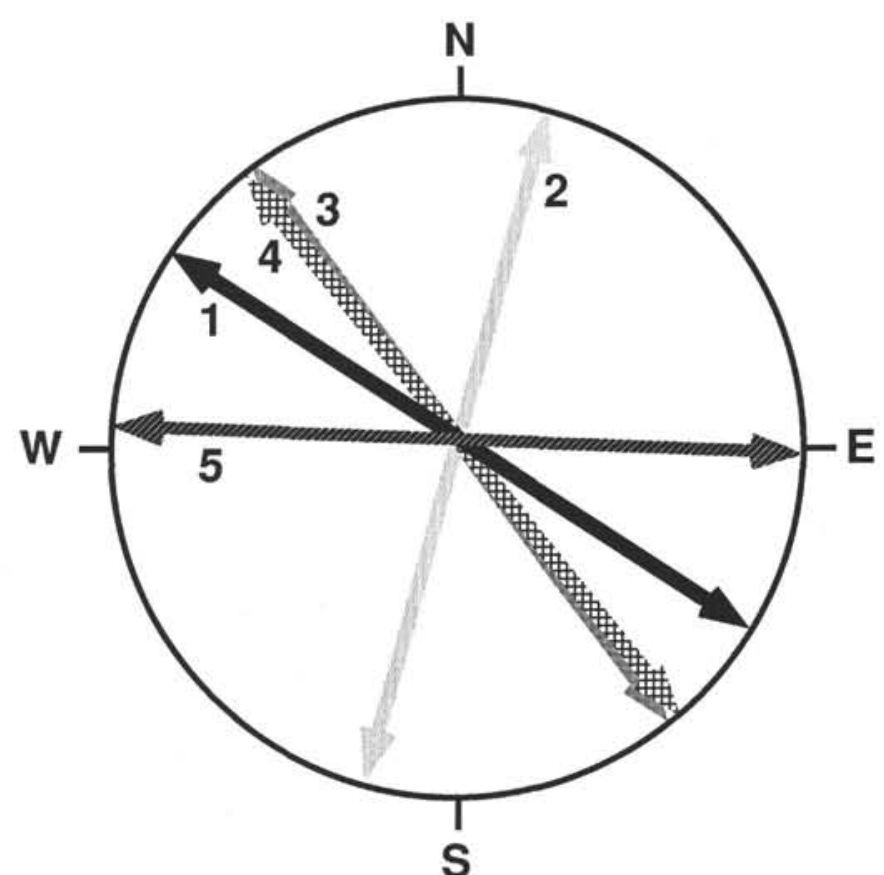

Figure 19. Summary of the fastest direction of $P$-wave propagation. 1, OBDS for inner circle shooting; 2 , JRT3; 3, JRT4; 4, RT6; 5 , ray path deviation.

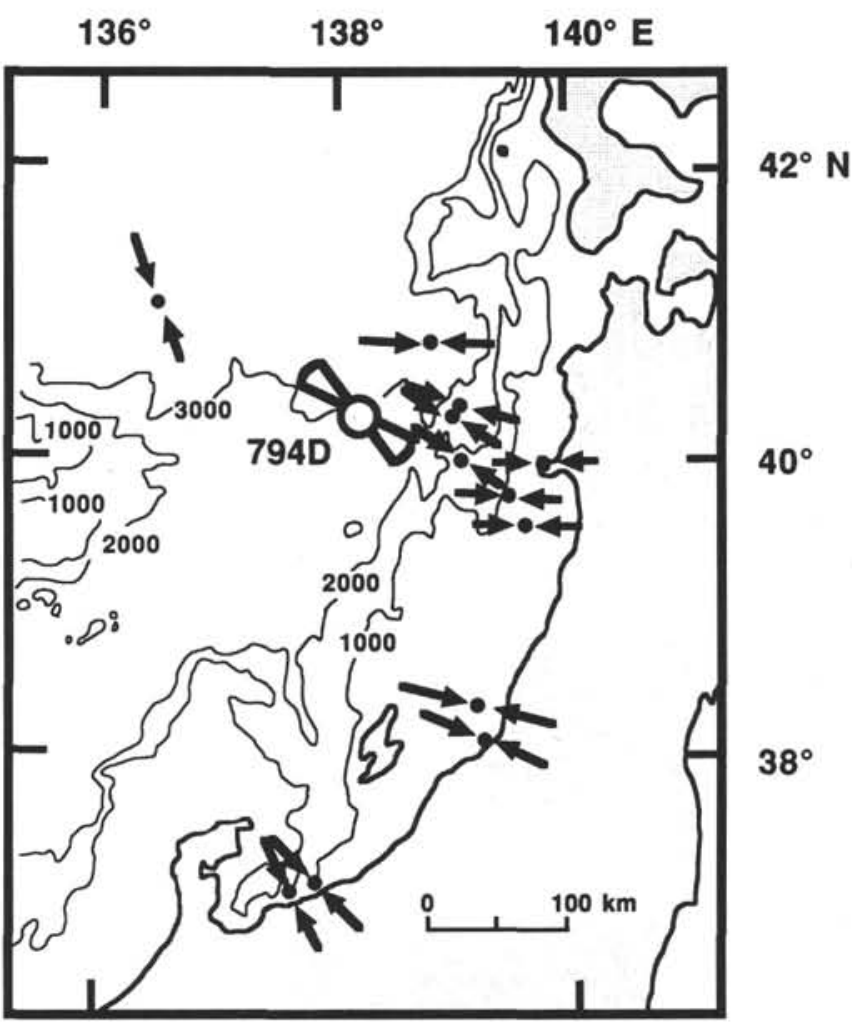

Figure 20. Regional stress field determined by studies of earthquake mechanisms (Yamazaki et al., 1985). Directions of the maximum compression are shown by arrowheads with dots at which earthquakes occurred. Probable range of the fastest direction of $P$-wave in the crust near Hole 794D is shown by fans. Bathymetry in meters. 
Without compression

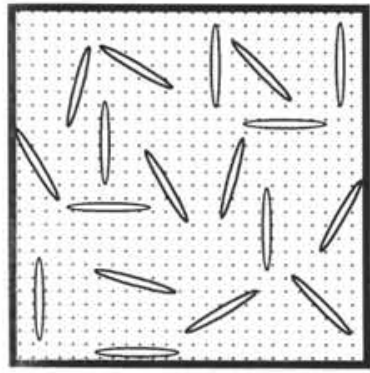

With compression

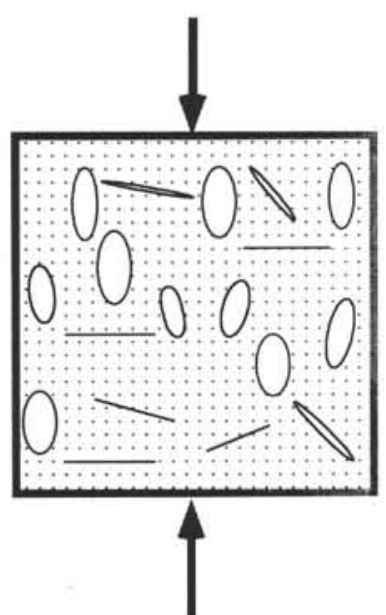

Figure 21. Schematic diagram showing stress-induced opening and closing of cracks with random orientations. Open cracks without preferred orientation (left) are effectively aligned due to compressive stress field in the direction of the maximum principal axis (right). 